Research Article

\title{
Intangible Cultural Heritage High-Definition Digital Mobile Display Technology Based on VR Virtual Visualization
}

\author{
Rongdong Xie \\ Guizhou University of Finance and Economies, Guizhou 550025, China \\ Correspondence should be addressed to Rongdong Xie; art123@mail.gufe.edu.cn
}

Received 19 May 2021; Revised 5 June 2021; Accepted 22 June 2021; Published 19 July 2021

Academic Editor: Sang-Bing Tsai

Copyright (c) 2021 Rongdong Xie. This is an open access article distributed under the Creative Commons Attribution License, which permits unrestricted use, distribution, and reproduction in any medium, provided the original work is properly cited.

\begin{abstract}
With the continuous improvement of the public's pursuit of information contact experience, in the era of the development of VR technology, digital mobile display technology has gradually changed the traditional display habits with its unique charm and strong competitive advantage. This article aims to improve the value of art research, meet the needs of the information age, and spread the spirit of intangible cultural heritage by improving the virtual visualization of intangible cultural heritage digital display technology. This article takes Dai ceramics intangible cultural heritage as an example to discuss the modern display technology of the ceramics, using virtual reality technology to enhance the sense of reality and interaction during the exhibition. This article uses visualization technology to digitally and artistically process Dai ceramics to achieve artistic effects while ensuring the authenticity of the ceramics, giving people a different visual experience. The article compares the application of several modern technologies in digital mobile display. Among them, the virtual reality technology has a good application in the system frame structure of the display, which exceeds $25 \%$. In the future, virtual visualization technology will be further popularized and promoted, giving intangible cultural heritage high-definition digital display more opportunities and development space.
\end{abstract}

\section{Introduction}

As the intangible cultural heritage enters the diversified era of information dissemination, virtual reality technology, as a new type of information technology, emerges at the historic moment. With incomparable advantages in information transmission, the display content is expanded to a higher dimensional virtual space. Through brand-new open technology and rigorous integration of information resources, traditional art can fully demonstrate the advantages of information resources, visual aesthetics, cognition and communication, and educational practice. These chained reality feedbacks stimulate the change of information display concepts and technological innovation in real time. Digital display based on virtual reality technology redefines the spatial language of traditional art display.

The development of society is inseparable from the promotion of production technology, and the development of the display design field is also inseparable from technological innovation. Nowadays, the intangible heritage art of the oldest traditional crafts is still preserved. As a representative technique of primitive art, the precious historical and cultural information contained in it makes it of high art scientific research value. For the display of intangible cultural heritage art, its traditional display form in the past has been unable to meet the era's demand for openness, freedom, and communication and interaction in information transmission.

More and more scholars have carried out research on intangible cultural heritage display technology. Liu X discussed the technical issues of developing $3 \mathrm{D}$ visualization independent software based on a virtual globe (Google Maps/Earth Geographic Information System (GIS)) for the navigation of small aircraft, with the purpose of promoting the safety and adequacy of nearby small aircraft. The system was developed using Web services and object-oriented programming disciplines to support the integration of virtual global frameworks, GPS, and real-time imaging data. He also developed a data processing program that glues C\# language, JavaScript language, National Marine Electronics Association (NMEA) instance data, and 
Keyhole Markup Language (KML) data together. The evaluation framework has been tested in nearby coastal areas. In order to evaluate the effectiveness of the system's performance and functions, he conducted a questionnaire survey among third-year students of Kobe University's navigation course. The evaluation of this system shows that the navigation assistance system for small aircraft has valuable potential. However, his research has experimental limitations, and the age of the survey subjects is limited [1]. Nurjanah developed the Aceh Disaster Digital Archive to make information about past disasters easier for the community. It uses an open source data platform, allows free access, and has interactive and easy-to-use features, which are essential to attract the younger generation. Similar disasters can happen anywhere in the world. By collecting multimedia data related to before and after the tsunami in alternative media, they present it in a visual form and associate it with social network services (SNS) to promote information about Asia and integrate information and knowledge of earthquake and tsunami experiences to promote sustainable disaster reduction. Information is the most important issue in disaster risk reduction (DRR). The transmission and dissemination of information are necessary, and it is sustainable from generation to generation. In 2004, the Indian Ocean tsunami occurred in Aceh. It is estimated that the tsunami caused more than 200,000 deaths. One of the main reasons is GAP information from past disasters. Today, information can be delivered and spread to all parts of the world as quickly as possible through the following methods. They realize the characteristics of information communication through the interactivity of virtual reality technology, but their research is not widely used in information media [2]. Zhou Y's research investigated the activities of the local intangible cultural heritage protection centers in Nanyang, Kaifeng, Xianning, Chibi, Sanming, and Jingdezhen and used semistructured interviews to record the current status of digital preservation of China's intangible cultural heritage, thereby investigating the field's status quo. The study found that the digital preservation system of Chinese cultural heritage has a clear structure and multiple components. These centers have abundant digital resources, but the storage is chaotic, and they are supported by policies, but the management field is weak. It is necessary to further clarify the rights and responsibilities of these institutions, formulate storage standards, establish a sharing platform, and strengthen digital preservation management. His research emphasized the protection of intangible cultural heritage, but did not clearly state the method of intangible cultural heritage protection [3].

The innovations of this article are as follows: (1) taking Dai pottery art as an example, it combines the digital threedimensional modeling of Dai pottery with the online grid and presents it with the characteristics of simple operability and real-time interactivity, achieving a complete virtual immersive display process; (2) combining art and technology to complement each other, streamline the structure of information transmission, shorten the time period of information transmission and feedback, improve the overall connotation of digital culture, and enhance the display depth of sensory experience.

\section{Research Methods of Intangible Cultural Heritage High-Definition Digital Mobile Display Technology Based on VR Virtual Visualization}

\subsection{Virtual Reality Technology}

2.1.1. The Meaning of Virtual Reality Technology. Virtual reality, referred to as $\mathrm{VR}$, is also known as Lingjing technology [4]. It is the applied technology concept involved in the digitalization argument of the Dai Pottery Museum in this subject [5]. As a new digital technology that began to emerge in the 20th century, it is the result of the combined development of computer display technology, computer graphics technology, sensing, artificial intelligence, and other fields for a long time. As a computer system that realizes corresponding operation feedback to human senses such as touch, hearing, vision, and balance, through complex calculations, VR is a human-computer interaction experience technology $[6,7]$. The specific application to the subject is to construct digital Dai pottery through digital means and then build a digital virtual environment that can be interactively operated to achieve a good display effect of Dai pottery art. It lets users get an immersive "physical" Dai pottery display experience in an artificially created environment [8]. The generation of this virtual reality environment is composed of several major elements such as users, computers, transmission equipment, and databases (as shown in Figure 1). It has outstanding characteristics such as multisensitivity, interactivity, nonsequence, and spatial malleability. It can create a virtual functional environment that meets people's multidimensional information needs through computers, simulating reality and trying to surpass reality. Not only that, the developing VR is also a very promising technology in the future, and it has very attractive application prospects in the fields of life, entertainment, education, or military industry.

2.1.2. Characteristics of Virtual Reality Technology. (1) More perceptual: multisensitivity refers to the perceptual characteristics of human senses such as vision, hearing, touch, smell, taste, and sense of balance. In virtual technology, it refers to the characteristics that can satisfy the user's biological perception function [9]. At present, due to technical limitations, the perceptual design in the human-computer interaction of the Dai Tao Digital Museum mainly stays in the main aspects such as vision and hearing. (2) Interactivity: it refers to the characteristics of communication and interaction between humans and computers in the virtual environment [10, 11]. Technical methods related to this feature include motion capture, tactile feedback, eye tracking, electromyography simulation, gesture tracking, direction tracking, voice interaction, sensors, and really venues (referring to the real scene partly consistent with the virtual world). The current design of Dai Pottery Digital Museum emphasizes entering the digital Dai pottery three-dimensional display environment through 
the computer interface and conducting interactive digital browsing with flexible mouse actions [12, 13]. (3) Nonsequential: it refers to the user's choice thinking in the experience of the virtual environment with certain jumping and nonsequential characteristics. Therefore, in the design simulation of the virtual environment of the Dai Pottery Digital Museum, the randomness of the user's choice will be taken into account, and the interactive design will also deepen the emphasis on the creation of feedback and naturalness. Compared with the traditional man-machine operating experience, this feature is the unique advantage of VR interaction. (4) Space ductility: it means that the transmission of information through network technology has broken through the geographical and time constraints of information exchange, and the space of human-computer interaction has gradually changed from $2 \mathrm{D}$ to $3 \mathrm{D}$, which greatly broadens and enriches users' thinking and operation in virtual space. Imagination is conducive to the improvement of interaction enthusiasm $[14,15]$. Therefore, the three-dimensional display of the Dai Pottery Digital Museum is also a breakthrough in a new "space" for Dai pottery art.

\subsubsection{Application of Virtual Reality Technology}

(1) Teaching. The advantage of VR display is that it can express objects and content vividly and clearly in virtual space, and users can obtain information feedback through communication and interaction in the environment. For example, putting VR in a teaching application can make users more intuitive and efficient to cultivate learning enthusiasm in a fun way $[16,17]$. For example, the VR virtual experiment platform can replace time-consuming and labor-intensive real experiments through computer simulation of virtual experiment objects. VR technology can also bring teachers and students to a simulated teaching classroom. Participants in the classroom can communicate in remote classrooms without being onsite. These are heights that are difficult to achieve in traditional education demonstrations. In the applications that have been developed, such as the multimedia teaching software of mechanical manufacturing engineering researched by Zou Xiangjun and Zhou Rongan, the virtual effect shown in its operation is very real $[18,19]$. The application demonstration has been waiting for good feedback from teachers and students in the command teaching of Nanhua University and National University of Defense Technology. The application of the Dai Ceramics Digital Museum is also an embodiment of the application of VR display in the popularization and education of traditional culture.

(2) Archaeology. At present, three-dimensional digital simulation technology has been used in archaeology to restore cultural relics with virtual data, thereby establishing a virtual database of three-dimensional digital cultural relics and historical sites $[20,21]$. The application in the Dai Pottery Digital Museum is also an efficient, high-precision, scientific digital storage of precious Dai pottery cultural resources and the permanent continuation of the actual meaning of traditional culture, and then digitally spread through the network platform to share and display the ceramic culture of the Dai people. Even staying at home, it can make history and culture "travel" every corner of the world.

\subsection{Research Methods}

2.2.1. Case Study Method. Observe and collect several existing digital museum cases, and refine and summarize the research situation at home and abroad. On the basis of analyzing the common law, guide the individual research of this topic [22].

2.2.2. Observation Practice Method. With the aid of the existing virtual reality technology research, the author taught myself the process of 3D model digital display-related technology and implemented it in the subject. On the basis of understanding a variety of digital display cases, the technical feasibility of the Dai Ceramics Digital Museum is analyzed and practiced [23].

2.2.3. Experience Summary Method. In the early stage of preparation for observation and preparation of Dai Pottery Digital Museum, the problems and thoughts encountered were collected theoretically, and the summary of the existing production experience was used as the preliminary preparation for the study of this article.

2.2.4. Investigation and Consultation Law. Random surveys and consultations were conducted on the relevant audiences of Dai pottery art and digital museum research, and then, the opinions were sorted and analyzed. Collect the feedback data of relevant users and apply them to the experience design research of the Dai Pottery Digital Museum.

2.3. Visualization Technology. The image edge detection technology is mainly based on the two-dimensional graphic image of the object for detection, and the edge feature line of the object in the image is determined through its related technology. The main object extracted by this type of method is the performance of the object's modeling feature on the two-dimensional plane $[24,25]$. The main information in the two-dimensional image containing the object exists at the edge of the image. The midgray level changes at the edge of the image are relatively drastic. Therefore, the edge is the boundary of the area where the gray level changes in the image, generally including step-like and roof-like shapes both types. The gray changes in the two sides of the stepshaped edge are relatively obvious, while the roof-shaped edge is at the junction of the gray value increase and the gray value decrease in the image. Therefore, the change of the edge point can be expressed in the form of the derivative, that is, the first derivative of the step-shaped edge and the second derivative of the roof-shaped edge are obtained [26]. Based on the derivative of the two, the result is that the first derivative of the step-like edge gray-scale change curve reaches a maximum here [27], and its second derivative intersects with zero here. The second derivative of the gray- 
scale variation curve of the roof-like edge reaches a maximum here, and its first derivative intersects with zero here [28].

Image filtering: calculating the first and second derivatives of image gray changes is the main content of image edge detection technology, and noise has a great influence on the calculation of derivatives. Therefore, filters are needed to work with edge detectors to ensure detection accuracy.

General filter function:

$$
T_{5}=\frac{1}{n} \sum_{i=0}^{n} T_{i} H_{i}
$$

Gaussian filter function:

$$
H(x, y)=\frac{1}{2 \pi \sigma^{2}} e^{-\frac{x^{2}+y^{2}}{2 \sigma^{2}}} .
$$

Laplacian Gaussian:

$$
\nabla^{2} H(x, y)=\left[\frac{x^{2}+y^{2}-2 \sigma^{2}}{\sigma^{4}}\right] e^{-\frac{x^{2}+y^{2}}{2 \sigma^{2}}} .
$$

Image enhancement: this step is to highlight the change of the neighborhood intensity value by strengthening the algorithm and then highlight the edge of the image. The change of the neighborhood intensity value is the basis of the edge enhancement display.

Gradient function:

$$
\frac{d g}{d x}=\lim _{\nu \longrightarrow 0} \frac{g(x+v)-g(x)}{v} .
$$

The modulus of the gradient is

$$
G[g(x, y)]=\left[\left(\frac{\partial g}{\partial x}\right)^{2}+\left(\frac{\partial g}{\partial y}\right)^{2}\right]^{\frac{1}{2}}
$$

Angle image function:

$$
\xi(x, y)=\arctan \left|\frac{\partial g / \partial y}{\partial g / \partial x}\right| .
$$

Image detection: determining the edge points of the image through the edge detection criterion is the gradient amplitude threshold criterion, so as to avoid the point's large gradient amplitude and mistakenly serve as the edge point.

The DoG (Difference of Gaussian) function is defined as the difference between the Gaussian kernel of different scales and the image convolution result:

$$
T(x, y, \tau)=K(x, y, l \tau)-K(x, y, \tau) .
$$

Key Point Descriptor in the template diagram:

$$
Q_{i}=\left(r_{i 1}, r_{i 2}, \ldots, r_{i 128}\right) .
$$

Descriptor of key points in the real-time graph:

$$
W_{i}=\left(w_{i 1}, w_{i 2}, \ldots, w_{i 128}\right) .
$$

Any two descriptors' similarity measure:

$$
t\left(Q_{i}, W_{i}\right)=\sqrt{\sum_{j=1}^{128}\left(r_{i j}-w_{i j}\right)^{2} .}
$$

Image positioning: point out the precise position and orientation of the edge through the resolution of the image subpixels. In this method, the first three steps are usually used. This is because in most cases, we only need to find that the edge appears in the vicinity of a certain pixel in the image and does not need precise position or direction.

Display image comprehensive evaluation index:

$$
P=\frac{\left(\chi^{2}+1\right) q * T}{\chi^{2}(q+T)} .
$$

When the parameter $\chi=1$ is the most common $P 1$, that is,

$$
P 1=\frac{2 * Q * T}{Q+T},
$$

a higher value of $P 1$ indicates that the method is more effective.

\section{Technical Model of Intangible Cultural Heritage High-Definition Digital Mobile Display Based on VR Virtual Visualization}

3.1. Overall Framework of the Intangible Cultural Heritage Digital Display Model. The research on the intangible cultural heritage high-definition digital mobile display technology in this section is mainly divided into three parts. The first part is based on the reverse engineering technology to digitally collect the selected Dai ceramics, perform data processing, and finally obtain a complete digital three-dimensional model; the second part is to use computer rendering software such as 3Dmax and KeyShot to render the real effect of the digitized three-dimensional model that has been obtained. The rendering requirements here are fine to restore the authenticity of the ceramics and let the audience feel the virtual reality technology. The third part is aimed at the artistic static display of ceramics. Through the computer plane effect processing software, the rendering effect pictures obtained before are artistically processed, so as to achieve the artistic effect while ensuring the authenticity of the ceramics and giving people a different visual experience. Figure 2 shows the technical route of intangible cultural heritage mobile digital art research. 
3.2. 3DSS Three-Dimensional Scan Truncation. Acquisition of ceramic data information: this article is based on reverse engineering technology. The instrument used is the standard 3DSS-STD-II three-dimensional scanner owned by the R\&D Center of the Industrial Design Department. This three-dimensional scanner is mainly based on structured light scanning to obtain object data. Information: the accuracy and precision of obtaining data information are relatively high.

The basic principle of the 3DSS three-dimensional scanner is that it is an instrument that uses structured light to scan. Because it has two cameras, it can acquire images of the same object from two angles at the same time and analyze the data information in the image. It is convenient for later application. After the collected image information is analyzed and sorted, the three coordinates of the pixel can be calculated by combining mathematical principles. Table 1 shows the performance indicators of 3DSS.

For the omni-directional scanning of Dai ceramics, it takes a certain amount of time because when scanning work, it is necessary to adjust the angle of the periodic scanning every time. Each angle must obtain data information. This multiangle data acquisition makes us a large amount of point cloud data that has been obtained, and due to their different perspectives, all point cloud data need to be integrated into the same perspective for processing, that is, a new three-dimensional coordinate system needs to be established, and then convert all the point cloud data into this coordinate system. The data is transformed into this coordinate system. This conversion process needs to be carried out according to the reference point, so we need to attach a certain size of reference point on the surface of the object when scanning. Since the ceramic used in this article is a 1:2 replica, its height is $6.5 \mathrm{~cm}$, its caliber is $14.95 \mathrm{~cm}$, and the scanning range is within $200 * 150 \mathrm{~mm}$, so a reference point with a diameter of $3 \mathrm{~mm}$ is selected.

\subsection{Image Processing Stage}

3.3.1. Filtering Strength. The setting of the parameter here is mainly to adjust the degree of smoothing of the obtained point cloud data. When the parameter value is adjusted higher, the surface details of the measured object are blurred, but the point cloud is smoother.

3.3.2. Boundary Trimming Width. During the overall scanning process, due to operating environment and other reasons, errors will inevitably occur. At the boundary where the point cloud data is discontinuous, the probability of occurrence is greater. The parameter settings here are used to correct these errors.

3.3.3. Automatic Corner Cutting. The scanning area generated by the scanner is usually a relatively neat rectangular area, and due to factors such as the placement of the lens and the operating environment, the scanning area displayed in the computer often produces a certain deformation. This kind of deformation will be a significant error in the measurement results, so here you need to select the automatic angle cutting setting to reduce the workload of subsequent repair errors.

CCD parameters are the default parameters used when scanning the reference point, so these parameters should be set according to the brightness of the reference point. The brightness is set to $10 \%$. The iris is always set to $15 \%$, and the default value of the gain is $10 \%$, but as the use time increases and the instrument wears out during use, the gain value parameter should be appropriately increased. Only in this way can the measurement accuracy be better guaranteed. In this subject, default values are used for parameter settings.

3.4. Data Processing Stage. The data processing stage is an important part of the entire reverse engineering work. Whether the operation of this step is reasonable will directly affect the subsequent work. This article uses Geomagic reverse engineering software in the data processing stage. The data collection of Dai ceramics uses a 3DSS three-dimensional scanner and uses a structured light measurement method to obtain point cloud data. In this way, what we obtain is a large amount of scattered point cloud data, so we need to use reverse engineering software for data to deal with.

\section{Intangible Cultural Heritage High-Definition Digital Mobile Display Technology Based on VR Virtual Visualization}

4.1. Intangible Cultural Heritage Digital Mobile Display Process. In this article, the Dai ceramics intangible cultural heritage culture is taken as an example, using virtual reality visualization technology for digital mobile display. First of all, we prepared a Dai ceramic hand-carved pot for model creation. By adjusting the point, line, and surface nodes, the reference object was simulated as close to the real as possible to achieve the most accurate and concise effect. Then, in 3DMAX, we performed UV unfolding on the surface texture of the model with a slightly complex structure and lined radian. This is to make the plane texture more accurately align the model, and the finished rendering of the final rendering is shown in Figure 3.

4.2. Comparison of Digital Mobile Display Effects. Taking Dai ceramic art as an example, the digital mobile display of Dai ceramics is compared with the traditional display. The comparison results are shown in Table 2. It can be clearly seen from the table that digital display can fully display the characteristics of intangible cultural heritage, exert its maximum comprehensive function and value, and improve the openness, fluidity, and virtual reality of information exchange.

The fixed place display method is widely used, which is another embodiment of the innovation in traditional display, and it also has the interaction of modern science and technology. The layout method is generally used in museum research and release sites, physical Dai pottery promotion booths, and Dai pottery teaching demonstrations. Generally, 


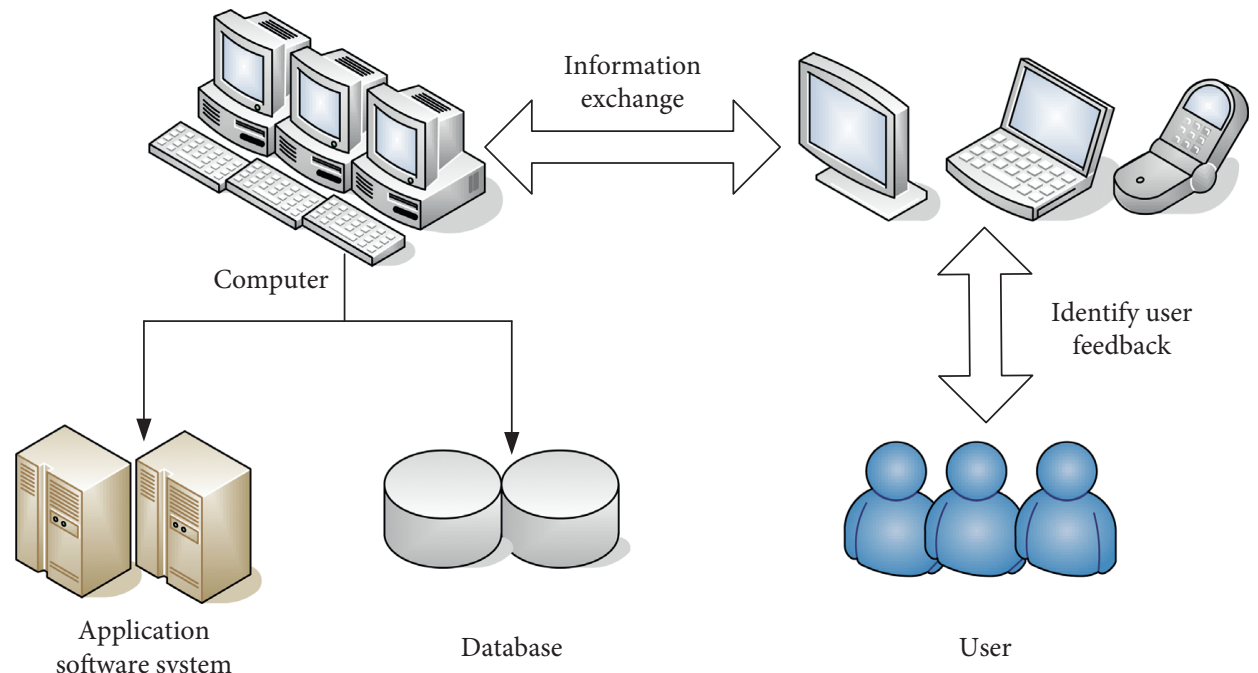

Figure 1: The main technical components of the virtual reality system.

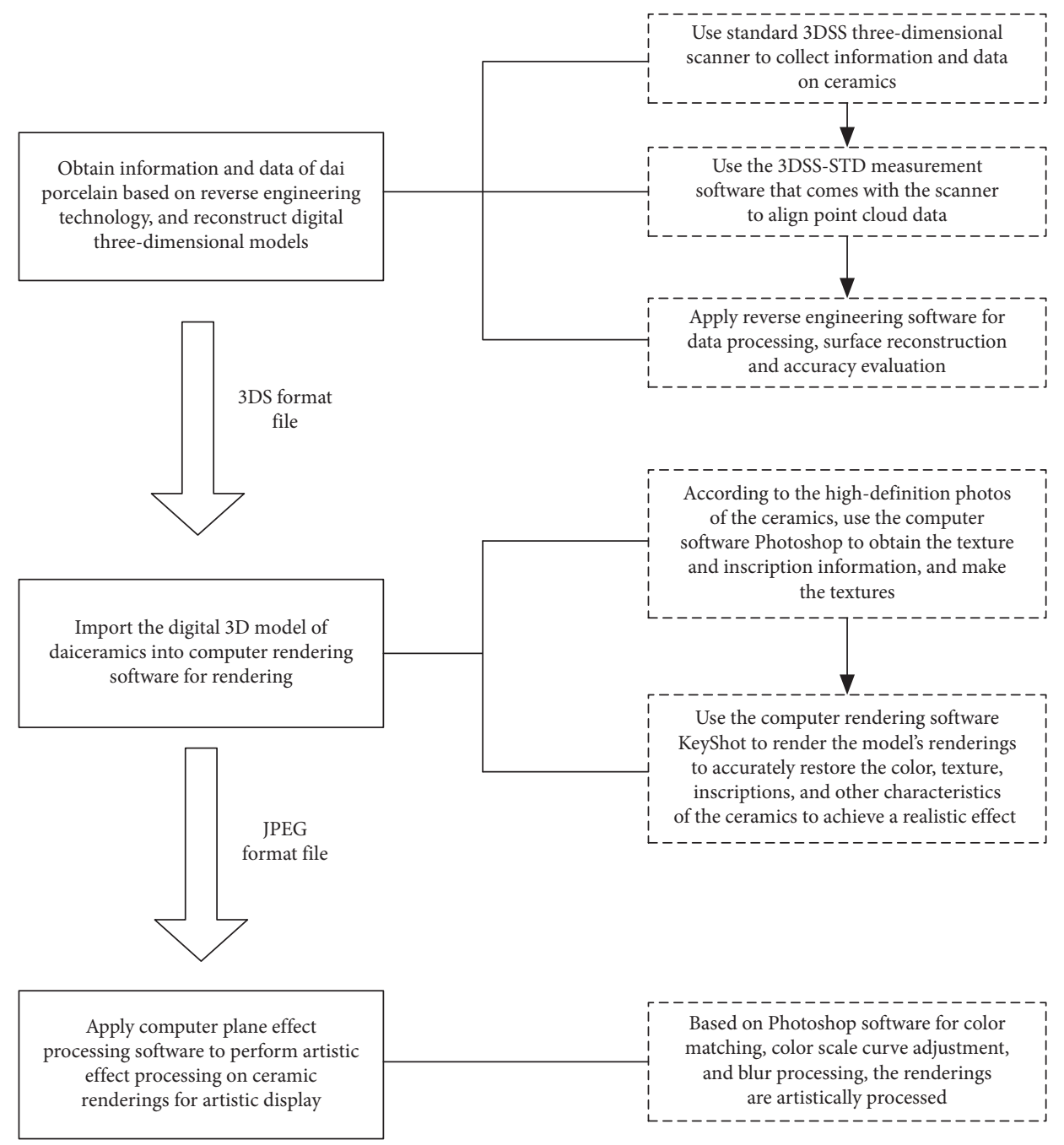

Figure 2: Digital mobile display technology roadmap. 
TABLE 1: 3DSS performance indicators.

\begin{tabular}{lc}
\hline Index & 3DSS-STD-II(standard type) \\
\hline Single scan range (typical value) & $400 * 300 \mathrm{~mm}$ \\
Single scan points & 130 \\
Color & --- \\
Scanning accuracy & $0.03 \mathrm{~mm}$ \\
Operating system & $\mathrm{Win} 2000 / \mathrm{XP}$ \\
Scan head weight & $5 \mathrm{~kg}$ \\
Single scan time & $5 \mathrm{~s}$ \\
Output file format & ASC, VRMl2.0, lgs, and stl \\
\hline
\end{tabular}

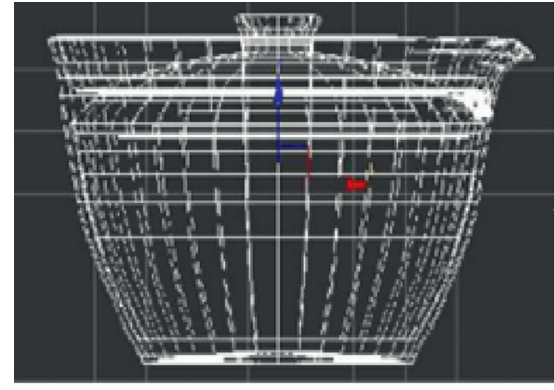

(a)

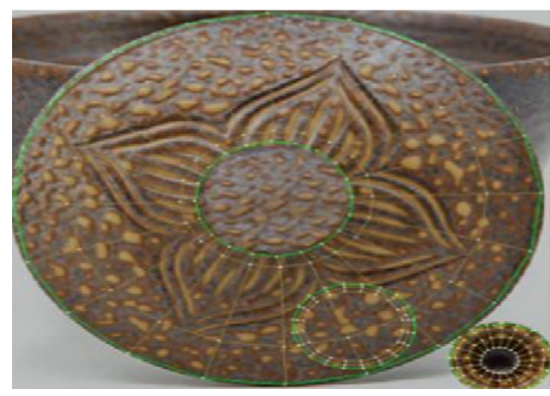

(c)

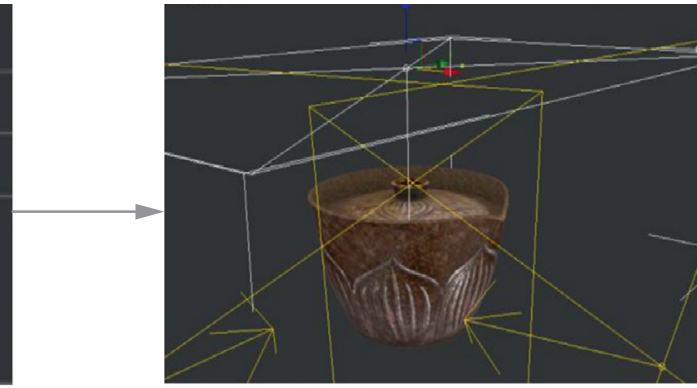

(b)

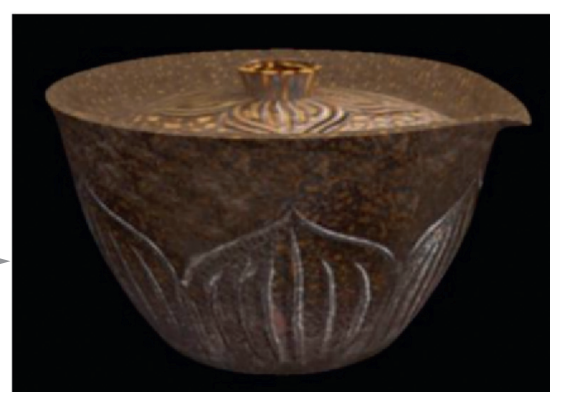

(d)

Figure 3: Model rendering process (the picture from Baidu Gallery). (a) Model creation. (b) Make a Texture. (c) UV unfolding (pot lid). (d) Rendered effect (overall).

suspended projection is used to project the picture onto the play screen or large electronic screen "virtual interactive explanation." Visitors can use it directly or watch the operation demonstration by multiple people. It can well drive the interactive atmosphere and produce to achieve the purpose of display.

Figure 4 is an interactive virtual display diagram of a fixed place. Placed in a specific place with a large number of people, the display effect is significant, and it also provides a place for physical communication between users and users or between users and designers, eliminates the transition time cost of virtual Dai pottery from recognizing the physical Dai pottery contact, and realizes the integration of publicity display and benefit output.

Figure 5 shows the application modules of modern technology. Virtual reality technology and augmented reality technology have similar applications. They are both widely used in video capture and positioning modules; there are fewer applications of tracking and registration principle technologies, which are used in system functions.
Architecture, video capture, and positioning module applications are all around 25\%.

4.3. Comparison of Data Acquisition of Virtual Reality Technology. Each data acquisition method has its own advantages and disadvantages. Table 3 shows a comparison chart of the advantages and disadvantages of several commonly used data acquisition methods. At present, several commonly used data acquisition methods are three-coordinate measurement method, laser triangle ranging method, structured light method, and CT method.

After scanning the image, a large amount of point cloud data is obtained, and the classification is shown in Table 4. We classify point cloud data from several perspectives such as disordered point clouds, noise points, and redundant points. This is a good measure of scanning equipment and scanning quality.

As can be seen from Figure 6, we compare the sampling rate of the scene point cloud signal according to the strength 
TABle 2: Comparison between traditional display and Dai ceramic digital display.

\begin{tabular}{|c|c|c|}
\hline $\begin{array}{l}\text { Property } \\
\text { comparison }\end{array}$ & Traditional display & Digital display \\
\hline \multirow[t]{2}{*}{$\begin{array}{l}\text { Natural } \\
\text { attributes }\end{array}$} & $\begin{array}{l}\text { Advantages: it has a sense of atmosphere and space for } \\
\text { display; the exhibition hall is organized and planned }\end{array}$ & $\begin{array}{l}\text { Advantages: you can experience the texture and physical } \\
\text { properties of ceramics on the spot; it is a symbol of brand } \\
\text { image and plays an important role in the source of } \\
\text { information }\end{array}$ \\
\hline & $\begin{array}{l}\text { Disadvantages: untouchable, time-sensitive considerations, } \\
\text { safety issues, and investment costs }\end{array}$ & $\begin{array}{l}\text { Disadvantages: the audience's own conditions are } \\
\text { restricted; the scale of the exhibition is independent }\end{array}$ \\
\hline \multirow{2}{*}{$\begin{array}{l}\text { Cultural } \\
\text { attributes }\end{array}$} & $\begin{array}{l}\text { Advantages: the display effect is highly pertinent; the display } \\
\text { categories are flexible and changeable; and, the information } \\
\text { blind spots are reduced }\end{array}$ & $\begin{array}{l}\text { Advantages: expand the openness and exchange of } \\
\text { information; improve comprehensive functions }\end{array}$ \\
\hline & $\begin{array}{c}\text { Disadvantages: the standards for digital information } \\
\text { transformation are not uniform; precious collections } \\
\text { cannot be copied }\end{array}$ & $\begin{array}{c}\text { Disadvantages: low social acceptance; digital intellectual } \\
\text { property development issues }\end{array}$ \\
\hline & $\begin{array}{r}\text { Both display methods are windows for the expression o } \\
\text { advantages that the traditional display form does not pos } \\
\text { reduces the damage rate of physical ex }\end{array}$ & $\begin{array}{l}\text { litional art and culture; the digital display form has the } \\
\text { does not occupy the resources of the original collection, } \\
\text { s, and promotes information exchange }\end{array}$ \\
\hline
\end{tabular}
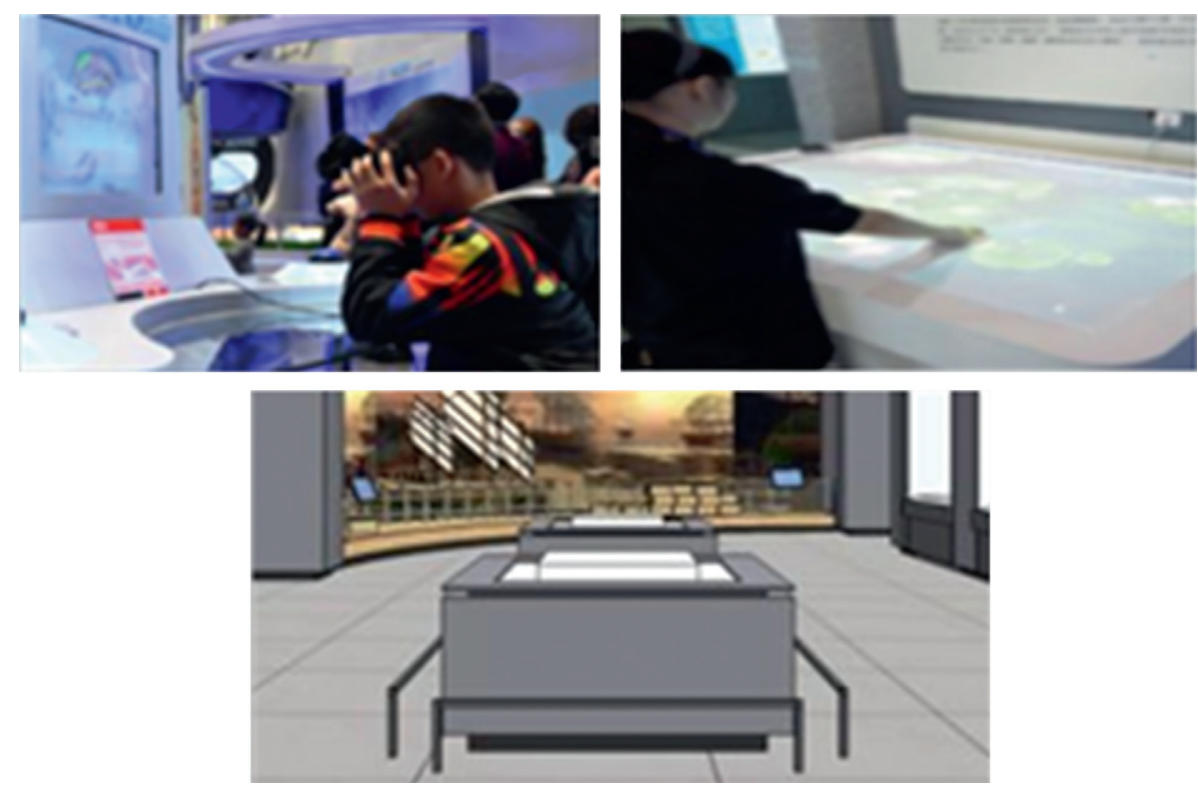

FIGURE 4: Interactive virtual display in a fixed place (the picture from Baidu Gallery).

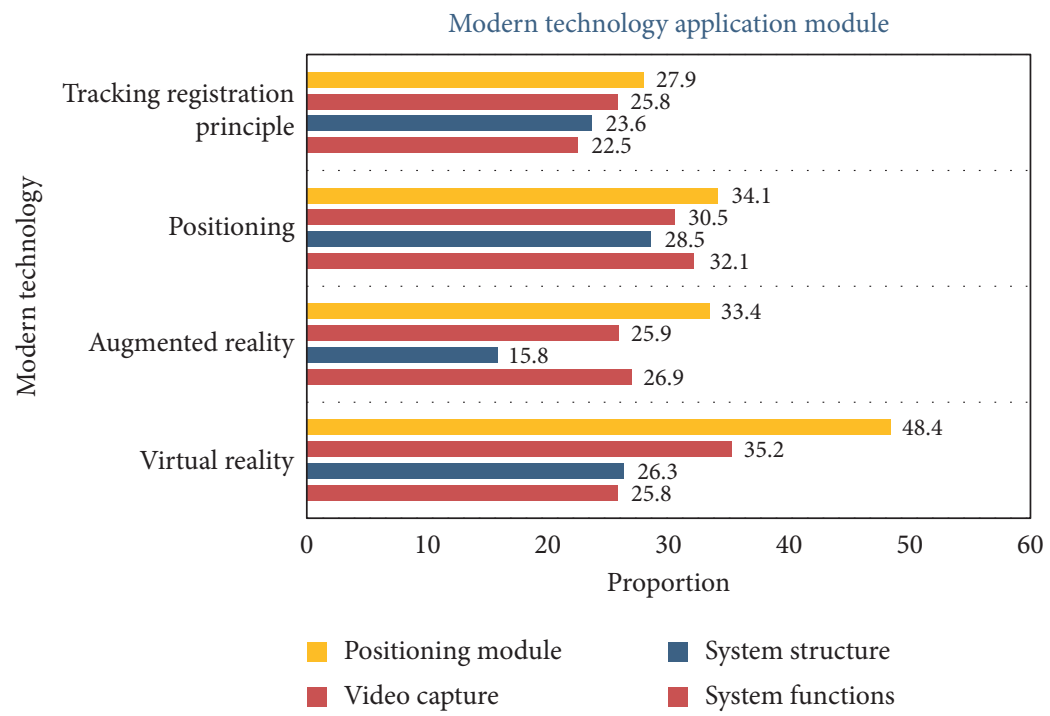

FIGURE 5: Modern technology application module. 
TABLE 3: Comparison of several data acquisition methods.

\begin{tabular}{|c|c|c|c|c|}
\hline Measurement methods & Three-coordinate measuring method & Laser triangular ranging method & Structured light & CT method \\
\hline Uncertainty & $0.6-30 \mu \mathrm{m}$ & $\pm 5 \mu \mathrm{m}$ & $\pm 1- \pm 3 \mu \mathrm{m}$ & $>1 \mathrm{~mm}$ \\
\hline Material restrictions & Limited & No & No & Limited \\
\hline Shape restrictions & No & The surface cannot be smooth & No & No \\
\hline Measuring speed & Slow & General & Fast & Slower \\
\hline Measuring cost & Higher & Higher & General & High \\
\hline
\end{tabular}

Table 4: Point cloud data classification.

\begin{tabular}{lcc} 
Scanning device & $\begin{array}{c}\text { Ordered point } \\
\text { cloud } \\
\text { Disordered point } \\
\text { cloud }\end{array}$ & Arrange at a constant density in the direction of rows and columns \\
Miscellaneous & $\begin{array}{c}\text { Miscellaneous points are points that are measured incorrectly and are invalid points; after } \\
\text { zooming in, you can see that they are clearly separated from the surface of the part and exist } \\
\text { in isolation }\end{array}$ \\
$\begin{array}{l}\text { Scan quality } \\
\text { Noise point }\end{array}$ & $\begin{array}{c}\text { Because of the reverse equipment and measurement method, there are systematic and } \\
\text { random errors in the measurement data; some of the measurement points have relatively } \\
\text { large errors that exceed our allowable range; this is the noise point }\end{array}$ \\
$\begin{array}{l}\text { Generated during } \\
\text { operation }\end{array}$ & Redundant point & $\begin{array}{c}\text { It is because of the problems of flattening, measuring angle, etc., that overlap redundant } \\
\text { points }\end{array}$ \\
\hline
\end{tabular}

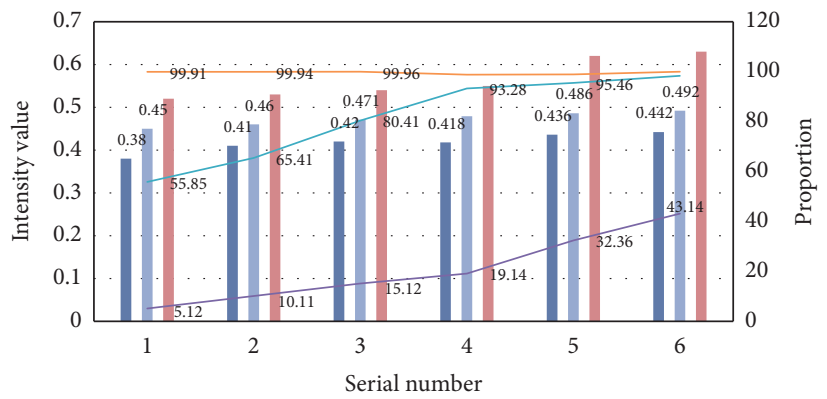

$$
\begin{aligned}
& \text { Collecting data from ceramic entities } \\
& \begin{array}{ll}
\text { Signal strength 1 } & - \text { Sampling rate 1 } \\
\text { Signal strength 2 } & - \text { Sampling rate 2 } \\
\text { Signal strength 3 } & - \text { Sampling rate 3 }
\end{array}
\end{aligned}
$$

Figure 6: Collecting data from ceramic entities.

of the callback signal. On the whole, the signal strength interval is controlled between $0.35-0.65$. When the echo signal strength is increased from 0.33 to 0.44 , the sampling rate is increased by $32.36 \%$. The laser echo signal area of intangible entities is in the range of $0.47-0.58$.

It can be seen from Figure 7 that the model proposed in this article can effectively shorten the loading time, especially when the viewpoint is closer to the cultural relics' exhibits, the optimization time is more obvious because the model that needs to be loaded at this time is relatively fine and the amount of data is large. It often exceeds the limited drawing time. To achieve the optimal picture quality and display time, the loading mechanism adopted in this article can ensure the best loading LOD level of the current frame and appropriately reduce the LOD level of some unimportant models to reduce the scene. Complexity improves the real-time performance of the system; when using a low-level LOD model, it can effectively increase the simplified quality of the model and ensure the best picture effect without exceeding the limited time so that users can browse cultural relics' exhibits from different perspectives in the scene and realize real-time roaming of virtual museum scenes. 


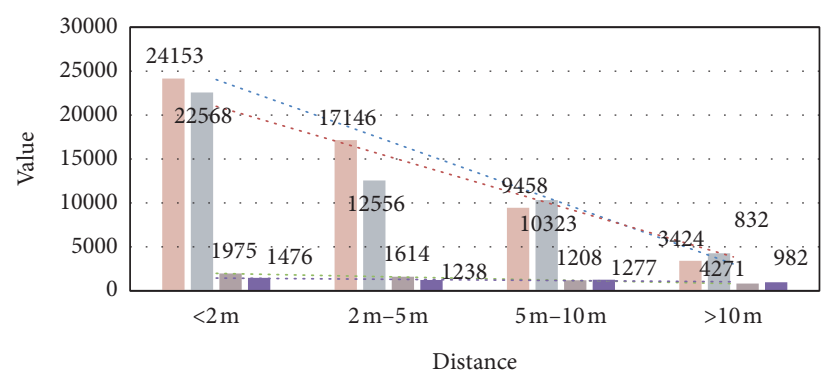

$$
\begin{aligned}
& \text { Scene load data } \\
& \text { The number of triangles before the scene is loaded } \\
& \text { The number of triangles after the scene is loaded } \\
& \text { Load time before optimization (ms) } \\
& \text { Optimized load time (ms) }
\end{aligned}
$$

FIgURE 7: Scene load data.

\section{Conclusion}

This article mainly researches the intangible cultural heritage high-definition digital mobile display technology based on VR virtual visualization. Through VR virtual visualization technology, the high-definition digital mobile display effect of intangible cultural heritage is improved, and the artistic effect and visual experience of mobile display are enhanced. The innovation of this article takes Dai pottery art as an example, combines Dai pottery digital three-dimensional modeling with online grid, and presents it with the characteristics of simple operability and real-time interaction, realizing a complete virtual immersive display process. This article is still in the process of three-dimensional digital display at the stage of theoretical and practical exploration. At present, there is still a certain degree of difficulty in the research on the transmission of the interest of the display information and the depth of the connotation. In terms of technical expansion and theoretical innovation, it takes time to further explore.

\section{Data Availability}

No data were used to support this study.

\section{Conflicts of Interest}

The author declares that there are no conflicts of interest regarding the publication of this paper.

\section{Acknowledgments}

The author received financial support from Scientific Research Project of Provincial Science and Technology Department, Guizhou Kehe foundation-ZK [2021] (provincial level), for the project titled "Research on Intangible Cultural Heritage Protection and Collation Based on Big Data and Artificial Intelligence.”

\section{References}

[1] X. Liu and S. Shiotani, "A virtual globe-based visualization and interactive framework for a small craft navigation assistance system in the near sea," Journal of Traffic and Transportation Engineering(English Edition), vol. 4, no. 6, pp. 68-79, 2017.

[2] H. Nurjanah, "Lesson learned from the Aceh tsunami of 2004: a digital multimedia display of the Aceh archive using an open-source platform for sustainable disaster risk reduction and global information," Dejitaru Akaibu Gakkaishi, vol. 2, no. 1, pp. 8-20, 2018.

[3] Y. Zhou, J. Sun, and Y. Huang, "The digital preservation of intangible cultural heritage in China: a survey," Preservation, Digital Technology \& Culture, vol. 48, no. 2, pp. 95-103, 2019.

[4] Z. Lv, X. Li, and W. Li, "Virtual reality geographical interactive scene semantics research for immersive geography learning," Neurocomputing, vol. 254, pp. 71-78, 2017.

[5] S. Tanaka and M. Hideo, "Introduction to the special issue on virtual reality and visualization for modeling and simulation," International Journal of Modeling, Simulation and Scientific Computing, vol. 9, no. 3, Article ID 1802001.1, 2018.

[6] K. Kang, S. Kushnarev, W. W. Pin et al., "Impact of virtual reality on the visualization of partial derivatives in a multivariable calculus class," IEEE Access, vol. 8, no. 99, 2020.

[7] Y. Peng, Y. Ma, Y. Wang, and J. Shan, "The application of interactive dynamic virtual surgical simulation visualization method," Multimedia Tools and Applications, vol. 76, no. 23, pp. 25197-25214, 2017.

[8] R. Yuan, Z. Zhang, P. Song et al., "Construction of virtual video scene and its visualization during sports training," IEEE Access, vol. 8, no. 99, p. 1, 2020.

[9] A. D. Obukhov, N. A. Chebotov, N. A. Chebotov, N. A. Vekhteva, K. I. Patutin, and N. N. Prokudina, "The development of a virtual installation visualization training system for oil and gas industry," Vestnik Tambovskogo Gosudarstvennogo Tehnicheskogo Universiteta, vol. 26, no. 4, pp. 571-580, 2020.

[10] N. E. Kalenov, S. A. Kirillov, I. N. Sobolevskaya, and A. N. Sotnikov, "Digital 3D-objects visualization in forming 
virtual exhibitions," Russian Digital Libraries Journal, vol. 23, no. 3, pp. 418-432, 2020.

[11] S. J. Buckley, K. Ringdal, N. Naumann et al., "LIME: software for 3-D visualization, interpretation, and communication of virtual geoscience models," Geosphere, vol. 15, no. 1, pp. 222-235, 2019.

[12] X. Zheng, H. Xiong, J. Gong, and L. Yue, "A morphologically preserved multi-resolution TIN surface modeling and visualization method for virtual globes," ISPRS Journal of Photogrammetry and Remote Sensing, vol. 129, pp. 41-54, 2017.

[13] S. Reddy and A. K. Singh, "Study on ocular morbidity prevalence and impact of digital display devices among school children," Indian Journal of Clinical and Experimental Ophthalmology, vol. 6, no. 3, pp. 413-417, 2020.

[14] X. Sang, X. Gao, X. Yu, S. Xing, Y. Li, and Y. Wu, "Interactive floating full-parallax digital three-dimensional light-field display based on wavefront recomposing," Optics Express, vol. 26, no. 7, pp. 8883-8889, 2018.

[15] I. Subri, A. Bayadir, T. Osama, and T. Abdulmuttalib, "Design and construction a falling water digital display system," International Journal of Computer Applications, vol. 177, no. 16, pp. 16-24, 2019.

[16] C. K. Hafstein and V. Tryggvi, "The flight of the condor: a letter, a song and the story of intangible culture heritage. 30 mins, DVD, colour Open access (vth@hi.is)," Journal of the Royal Anthropological Institute, vol. 25, no. 3, pp. 646-647, 2019.

[17] Y. Xiong and M. Lee, "Research on the symbiosis and coevolution of intangible cultural heritage tourism of rural traditional skills -- taking shuigoutou village as an example," The Journal of Asian Studies, vol. 23, no. 4, pp. 323-334, 2020.

[18] D. Drápala, "Social capital and safeguarding of intangible cultural heritage: theoretical concepts of departure and the potential of further research," Slovenský Národopis/Slovak Ethnology, vol. 68, no. 2, pp. 122-135, 2020.

[19] A. Raimkulova, "Actual problems OF conservation OF the intangible cultural heritage IN the context OF eurasian integration," Reports, vol. 4, no. 332, pp. 32-38, 2020.

[20] D. Sorea and C. Csesznek, "Intangible cultural heritage of the fagaras land. Priority dimensions," Bulletin of the Transilvania University of Brasov, vol. 13, no. 62, pp. 155-164, 2020.

[21] C. Smith and S. Bansal, "Intellectual property, cultural property and intangible cultural heritage," Antiquity, vol. 93, no. 372, pp. 1694-1695, 2019.

[22] K. Y. Hyun and G. H. Lee, "Analysis of change of event related potential in escape test using virtual reality technology," Biomedical Science Letters, vol. 25, no. 2, pp. 139-148, 2019.

[23] T. N. Chen, X. T. Yin, and X. G. Li, "Application of 3D virtual reality technology with multi-modality fusion in resection of glioma located in central sulcus region," Virtual Reality and Robotics in Medicine, vol. 98, no. 17, pp. 1302-1305, 2018.

[24] L. Lu, J. Ma, and S. Qu, "Value of virtual reality technology in image inspection and 3D geometric modeling," IEEE Access, vol. 8, no. 99, p. 1, 2020.

[25] L. V. Shalkevich, "Effectiveness OF virtual reality technology use IN complex rehabilitation OF manual activity IN children with cerebral palsy," Journal of the Grodno State Medical University, vol. 18, no. 6, pp. 716-721, 2020.

[26] N. Meng, "Application of intelligent virtual reality technology in Clothing virtual wear and color saturation after COVID-19 epidemic situation," Journal of Intelligent and Fuzzy Systems, vol. 39, no. 4, pp. 1-9, 2020.

[27] X. Li, J. Ling, Y. Shen et al., "Effect of color temperature of light source in tunnel on driving safety based on virtual reality technology," Tongji Daxue Xuebao/Journal of Tongji University, vol. 49, no. 2, pp. 204-210+288, 2021.

[28] Y. Wang and E. Liu, "Virtual reality technology of multi UAVEarthquake disaster path optimization," Mathematical Problems in Engineering, vol. 2021, no. 8, 9 pages, Article ID 5525560, 2021. 